\title{
Review
}

Journal of Innate

Immunity

\section{Gene-Specific Epigenetic Regulation in Serious Infections with Systemic Inflammation}

\author{
Charles E. McCall ${ }^{a} b \quad$ Barbara Yoza ${ }^{b, c}$ Tiefu Liu ${ }^{b}$ Mohamed El Gazzar ${ }^{b}$ \\ ${ }^{a}$ Translational Science Institute, ${ }^{b}$ Section on Molecular Medicine, Department of Internal Medicine, \\ 'Department of Surgical Sciences, Wake Forest University School of Medicine, Winston-Salem, N.C., USA
}

\section{Key Words \\ Facultative heterochromatin - Gene-specific reprogramming $\cdot$ Septic shock $\cdot$ Leukocytes $\cdot$ NFKB RelB $\cdot$ Histone code $\cdot$ Nucleosome positioning}

\begin{abstract}
Inflammation is a fundamental biologic process that is evolutionally conserved by a germ line code. The interplay between epigenetics and environment directs the code into temporally distinct inflammatory responses, which can be acute or chronic. Here, we discuss the epigenetic processes of innate immune cells during serious infections with systemic inflammation in four stages: homeostasis, incitement, evolution, and resolution. We describe feed-forward loops of serious infections with systemic inflammation that create gene-specific silent facultative heterochromatin and active euchromatin according to gene function, and speculate on the role of epigenetics in survival.
\end{abstract}

Copyright $\odot 2010$ S. Karger AG, Basel

\section{Introduction}

The typical features of inflammation, both good and bad, were recorded in the earliest human writing [1], which also recognized that no matter what injury or in- vading microorganism incited the process, an orderly sequence of events evolved. In fact, epigenetic changes written into the germ line explain how organisms generate different biologic responses to varying environmental factors and stress - in embryonic development, adult cell renewal, and specific diseases, such as cancer [2]. The importance of the interplay of germ line DNA and epigenetics in inflammation is still emerging [3].

The phases of inflammation differ contextually and temporally to generate the distinct host responses and outcomes that distinguish inflammatory diseases. For example, the systemic inflammatory response syndrome (SIRS) proceeds in a continuum from mild to severe forms of sepsis (or noninfectious causes) with vascular shock and/or multiorgan failure [4]. Serious infections kill millions yearly, with an unyielding mortality rate of $20-70 \%$ [5]. We refer to the phenotypes of serious infections as severe systemic inflammation (SSI). Here, we review the epigenetic gene-specific reprogramming, in the context of the unique pathophysiology of SSI. Readers are referred to a recent detailed review of transcription events controlling inflammatory genes, which alludes to epigenetics [6].

Several thousand innate immunity and inflammation genes may participate directly or indirectly in acute and chronic inflammatory processes [7]. In the basal (or homeostatic) state, they are repressed until a diverse recep-

\section{KARGER}

() 2010 S. Karger AG, Basel

Fax +41613061234

E-Mail karger@karger.ch

www.karger.com
Accessible online at: www.karger.com/jin
Dr. Charles E. McCal

Translational Sciences Institute, Wake Forest University School of Medicine Medical Center Blvd.

Winston-Salem, NC 27157 (USA)

Tel. +1 336716 2691, Fax +1 336716 1214, E-Mail chmccall@wfubmc.edu 


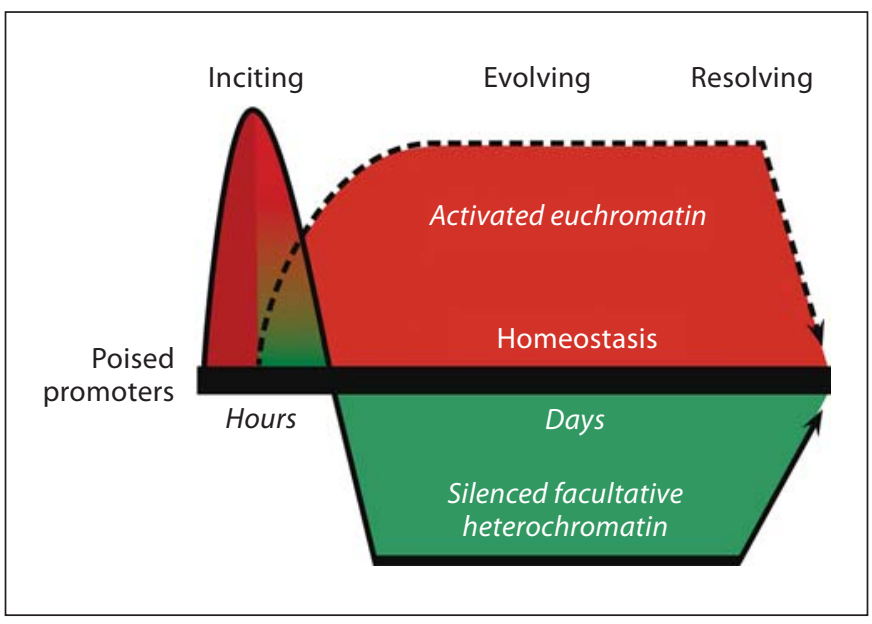

Fig. 1. Gene reprogramming during serious infection with SSI. Gene expression during SSI is depicted as occurring in four temporally distinct phases: poised; incitement; evolution; resolution. Following derepression of poised promoters, induction of rapidresponse proinflammatory genes incites an acute proinflammatory phase, which is rapidly terminated by posttranslational processes. The evolving phase is characterized by activated or repressed gene expression, according to function: rapid response proinflammatory genes that incited SSI are transcriptionally repressed by formation of facultative heterochromatin, and antiinflammatory and antimicrobial genes are activated from a euchromatin state. A return to homeostasis with reversal of facultative heterochromatin occurs during the resolving phase.

tor system, primarily the Toll-like receptors (TLRs), senses a threat [8]. It signals threatened cells to rapidly transcribe a set of poised proinflammatory genes to incite the acute phase of inflammation [9]. If the threat is limited, the acute proinflammatory genes return to the basal state within hours, and physiologic resolution follows [3]. If the threat is severe, the incitement phase is replaced within $4-6 \mathrm{~h}$ by a gene-specific epigenetic reprogram that may last from days to weeks; we observed it in humans for up to 21 days [10]. During this stage, certain gene modules (e.g. rapid-response, acute proinflammatory) are transcriptionally silenced, while others (e.g. anti-inflammatory, antimicrobial) remain activated [11]. This predictable and sustained epigenetic paradigm produces a distinct SSI clinical phenotype and predicts poor outcome [12].

The silencing reflects the phenomenon of endotoxin tolerance that correlates with high mortality and morbidity, but may or may not be directly causal for this. Its rapid development probably explains the failure of multiple anti-inflammatory therapies in human sepsis, which were given after the inciting phase [3], since epigenetics provides quick, natural protection against the excessive response and autotoxicity of acute pro-inflammatory gene products disseminated by the circulation. Note that the epigenetic silencing state apparently does not exist or, at least, persist during chronic inflammations like rheumatoid arthritis or regional enteritis [3], which, unlike SSI, benefit from anti-inflammatory therapies [13]. The reason for this paradox is unknown, but it has important therapeutic implications. We propose that distinct epigenetic mechanisms regulate acute and chronic inflammation.

Both clinical experience and systems biology provide useful contextual and temporal models for the gene reprogramming associated with SSI. We used both approaches to generate a scheme to elucidate SSI epigenetics (fig. 1). It reflects feed-forward loops directed by nuclear factor $\kappa \mathrm{B}(\mathrm{NF} \kappa \mathrm{B})$ members p65 and RelB, discussed in detail below. Our SSI model is divided into four phases: (1) basal homeostatic control, or poise; (2) release of the poised state with concomitant transcription of rapid-response genes that induce SSI, or incitement; (3) gene-specific epigenetic reprogramming that silences facultative heterochromatin while maintaining modules of activated euchromatin, or evolution, and (4) reversing gene-selective reprogramming and returning to homeostasis, or resolution. We acknowledge its simplistic nature. For example, it excludes the posttranscriptional events that control mRNA processing and protein synthesis, both of which are also directed by epigenetic events that control expression of RNA binding proteins and inhibitory RNAs [14]. It is still useful for correlating epigenetic processes with distinct chromatin modifications. Across this spectrum, sets of genes are silenced, expressed, or unperturbed, irrespective of inducing receptors and signaling pathways, emphasizing the ultimate control of specificity by chromatin $[3,15]$.

\section{Three Concepts Are Fundamental to Understanding SSI: Homeostasis, Feed-Forward Loops and Cell Survival Strategies}

\section{Homeostasis}

SSI is an extreme deviation from homeostasis, almost suggesting that evolution made a serious error. However, the evolutionary intent of all inflammation, including SSI, is to defend against attack and to return to a peaceful state. No one better articulated the homeostatic disaster of SSI than Lewis Thomas in his essay on The Lives of a 
Cell [16]: 'It is the information carried by germs that we cannot abide. Macromolecules are read by our tissues as the very worst of bad news. We are likely to turn on every defense at our disposal; we will bomb, defoliate, blockade, seal off, and destroy all the tissues of the area. It is shambles.'

Since homeostasis, as defined is dynamic, it is not surprising that opposing forces influence inflammation. Although this review focuses on nuclear-based epigenetic processes accompanying SSI, the coupling of cells to the environment during all types of inflammation depends on positive and negative regulatory processes in a variety of cell types [17]. During all phases of SSI, both positive and negative balancing mechanisms involve extra- and intracellular sensors, cytosolic and nuclear signaling pathways, and chromatin structural modifications. Extranuclear controls are not covered in this review.

\section{Feed-Forward Loops}

Both inductive systems biology and reductive reasoning emphasize the importance of feed-forward loops linked to the germ line and epigenetic codes of biologic processes, including inflammation. Systems biology identifies feed-forward loops using a hypothesis-generating approach [18], a tactic that unexpectedly identified activated transcription factor 3 (ATF3) in an NFкB-dependent repression loop in TLR-stimulated macrophages [19]. Feed-forward loops occur in several forms [20]. Using reductive research, we identified two epigenetic loops in SSI. One is an incoherent feed-forward loop in which an activator generates a primary target and its repressor. In SSI, p65 activator targets tumor necrosis factor- $\alpha$ $(\mathrm{TNF} \alpha)$ and interleukin-1 $\beta$ (IL-1 $\beta$, representing a larger module) and feeds forward to induce RelB as a target repressor [21]. The other is a coherent feed-forward loop in which an activator generates a primary target module as well as an activator of a distinct target. In SSI, the activator p65 targets TNF $\alpha$ and IL-1 $\beta$ and feeds forward to induce RelB as an activator of a distinct target module, e.g. IкB inhibitor- $\alpha(\mathrm{I} \kappa \mathrm{B} \alpha)$ [22]. Thus, these two feedforward loops from $\mathrm{p} 65$ generate RelB as a dual-function mediator of distinct targets with different functions and provide one essential mechanism for gene-specific programming that requires integrating transcription factors with epigenetic histone and DNA modifiers. In this context, the incitement phase generates both the cytokine burst and the epigenetic-dependent and gene-specific feature of evolving SSI. Resolution occurs when unknown forces reverse these loops. Other distinct loops undoubtedly exist, coupled or not to the p65-to-RelB loops. Systems biology provides an excellent strategy to identify them [23].

\section{Survival}

The evolutionary intent of life is survival; as a fundamental biologic process, inflammation must aim to support survival. How rescue loops link SSI epigenetics to survival is unknown, but the resolution state correlates with survival and a return to homeostasis. We speculate that the balance between death (apoptosis, necrosis) and life in cells and tissues during SSI links epigenetics with cell energy and nutrition in the attempt to repair injury and survive the shambles.

\section{The Basal State of SSI-Inducing Genes Is Epigenetically Silent but Poised}

As is schematically shown in figure 2, distinct combinations of protein, histone and DNA modifiers acting on proximal promoters direct the phenotypic phases of SSI.

Most rapid-response primary-response genes constitutively bind RNA polymerase II at their proximal promoters and, in some cases, must elongate or splice mRNA to initiate a higher level of transcription $[24,25]$. Recent information indicates that innate immunity genes are epigenetically repressed in the basal state but poised to respond rapidly to threats [26]. This homeostatic readiness involves a DNA complex that constitutively recognizes transcription factors c-jun or NFкB p50 as homodimers. They couple to nuclear receptor corepressor (NCoR), silencing retinoid acid mediators and thyroid hormone receptors (SMRT) [9].

In studies of TNF $\alpha, I L-1 \beta$, and I $\kappa$ B $\alpha$, we added an epigenetic layer to the poised-promoter concept $[22,27]$. Like most promoters of rapid-response, NFкB-dependent genes, the TNF $\alpha$ and IL- $1 \beta$ promoters constitutive-

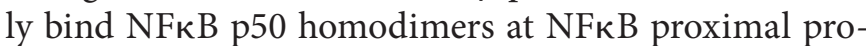
moter sites. A detailed analysis of the TNF $\alpha$ gene showed concomitantly bound histone $\mathrm{H} 3$ lysine 9-methyltransferase G9a, high-mobility box B1 (HMGB1) chromatin structural protein, heterochromatin protein 1 (HP1), and DNA cytosine methyl transferase DNT3a/b [27]. This functional complex directs dimethylation of histone $\mathrm{H} 3$ lysine 9 (H3K9) (a histone code silencing mark) and repressive hypermethylation of the adjacent DNA cytosine plus guanine $(\mathrm{CpG})$ sites. Conspicuous by its absence is histone $\mathrm{H} 1$ linker binding, which is a mark of more highly condensed chromatin [28]. Further, we find that in the basal state, a nucleosome covers the most active NFKB 
Fig. 2. Distinct combinations of protein, histone and DNA modifiers acting on proximal promoters direct the phenotypic phases of SSI (component details are provided in the text). Poised: a complex of p50 homodimers, transcription corepressors and epigenetic histone-silencing modifications combine in a checkpoint to prevent constitutive transcription. Inciting: TLRdependent processes derepress the promoters of rapid-response genes, leading to p65/p50 transactive heterodimer formation and competent transcription. Not shown is the rapid disassembly of the p65/ p50 heterodimer by p65 proteosome degradation (see fig. 1 and text). Evolving: A p65-dependent feed-forward loop induces RelB de novo, after which it both initiates gene-selective facultative heterochromatin formation to silence acute proinflammatory gene promoters and activates antiinflammatory gene promoters. Resolution is not shown but includes diminishing RelB production and reversal of chromatin modifications.

Fig. 3. Nucleosomes reposition at the TNF $\alpha$ promoter during SSI. The proximal promoter, about $-500 \mathrm{bp}$, is depicted, showing the $\mathrm{K} 2$ and $\mathrm{K} 3$ (active) $\kappa \mathrm{B}$ regulatory cis elements. During the poised state, nucleosome 1 covers K3. The LPS TLR4 inciting phase directs p65 binding and repositions nucleosomes 1 and 2 by a Nap1 histone 2A.Z chaperone. The RelB-initiated evolving phase repositions nucleosome 2 over the $\mathrm{K} 3 \mathrm{kB}$ site, where it remains after TL4 stimulation (endotoxin tolerance). The interdependent, multicomponent silencing complex that condenses chromatin by histone and DNA methylation is sustained as facultative heterochromatin during SSI evolution. RelB removal by siRNA repositions nucleosome 2 by ATPdependent BAF complex. It is not known how naturally resolving SSI repositions nucleosomes to generate a competent promoter.
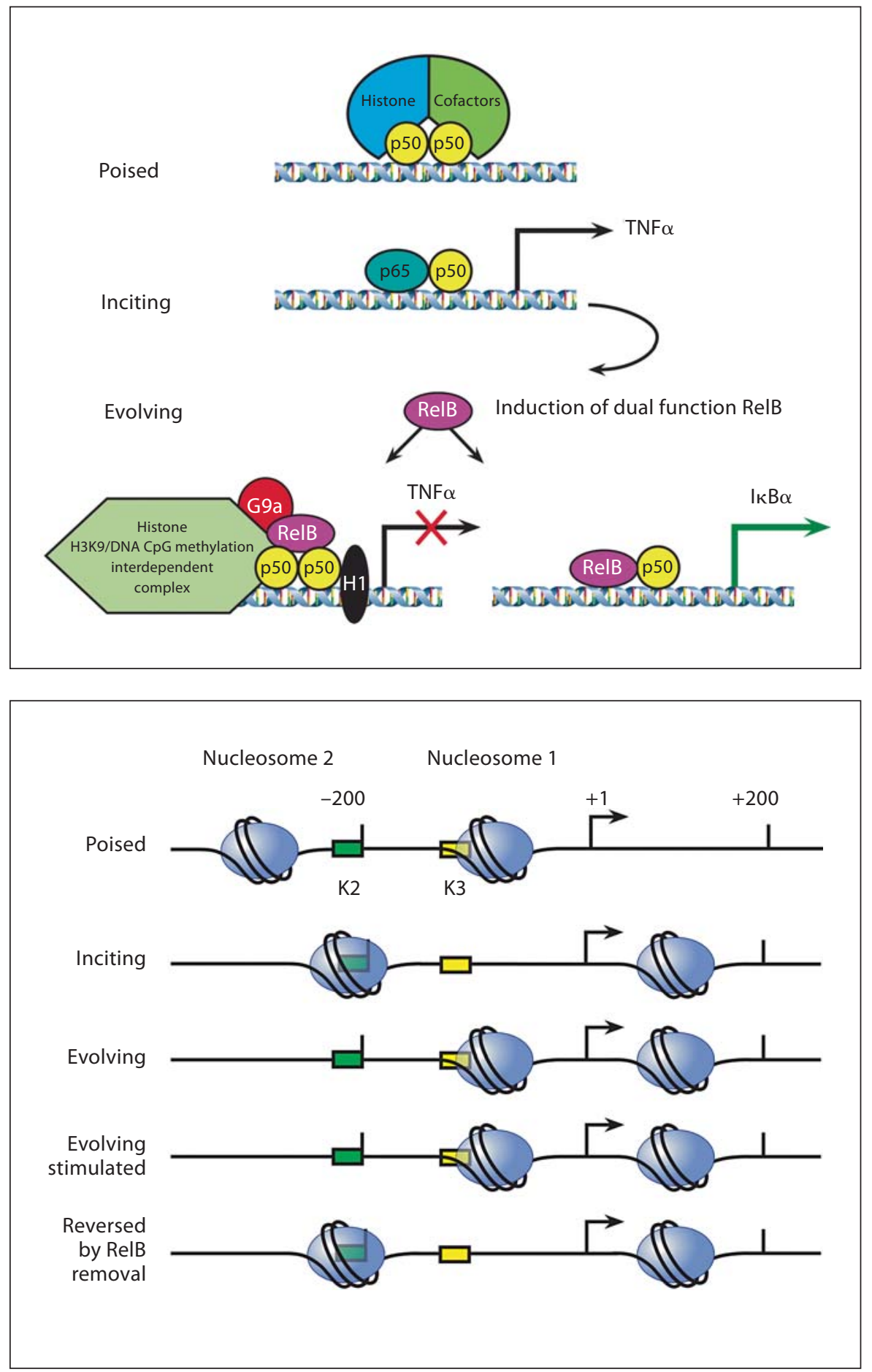

site (K3) [29], but its position differs in the evolution phase (fig. 3, discussed below).

Together, data support the conclusion that the poised state of the rapid-response genes responsible for inciting inflammation is epigenetically regulated homeostasis.
These gene-specific promoters are likely germ line encoded and emerge epigenetically during cell development and differentiation. For example, the TNF $\alpha$ promoter is highly CG methylated and inaccessible to NFKB activation in 3T3 murine embryo-derived fibroblasts, but environmen- 
tal changes can manipulate it to become responsive to lipopolysaccharide [30]. Thus, epigenetic check points for inflammation are specific to cell-type and environment.

\section{The Phase That Incites SSI Releases the Poised and Silent Epigenetically Controlled Basal State to Produce a Damaging Cytokine Storm That Is Rapidly Muted by the Disruption of Transcription}

This phase of SSI (fig. 2) has received the most attention since the discovery of the TLR innate immunity sensor system. Highly vigilant pattern recognition receptors, such as the TLR family, rapidly deliver bad news through a network of cytosolic and nuclear signals of limited specificity, as the ultimate specificity of the response is genedependent and epigenetically controlled [3,31]. As incitement begins, poised and epigenetically silenced acute proinflammatory genes derepress, allowing trans-acting factors to couple to cognate cis elements [9]. Any TLR that senses a threat can modify chromatin to link the poised promoters to mediator complex and RNA polymerase II. During this phase, the repressor complex is removed, and the active euchromatin state of demethylated H3K9, phosphorylated $\mathrm{H} 3 \mathrm{~S} 10$, and hypomethylated CpG promoter DNA occurs $[27,32]$. The precise mechanisms controlling derepression remain unclear but include ubiquitination and proteosome degradation $[33,34]$.

Whether SSI or the milder SIRS follows the infectioninduced incitement phase is ultimately determined by the magnitude of the threat and the resulting cellular and tissue-determined gene expression level [35]. All TLR sensors can probably induce SSI [36].

In the animal kingdom, the NFKB family is the master switch for inciting acute and chronic inflammation [37]. Among these members - RelA/p65, RelB, c-rel, p105/p50, p100/p52 - RelA/p65 (p65) is the commanderin-chief. Virtually all external (e.g. extracellular pathogens or alarmins) or internal (e.g. DNA breaks or intracellular pathogens) threats, once sensed, pass through the p65/p50/IкB cytoplasmic checkpoint, although other pathways (e.g. stress kinases and phosphatases) contribute. After cytoplasmic release by ІкB $\alpha$ proteosome degradation, cooperative mediators specifically modify p65 into its bound, transactive state by site-specific phosphorylation, acetylation, and methylation. An $\mathrm{H} 3$ serine kinase phosphorylates $\mathrm{H} 3 \mathrm{~S} 10$ to support chromatin opening and start-site competence [2]. Our unpublished observations support mitogen-activated stress kinase (MSK), IкB kinase- $\alpha$ (IKK $\alpha$ ), and/or Aurora B as possible regulators of this $\mathrm{H} 3 \mathrm{~S} 10$ phosphorylation. Whether chromatin opening first involves p65, the histone $\mathrm{H} 3 \mathrm{~S} 10$ kinase, or another process, such as $\mathrm{H} 3 \mathrm{~K} 4$ trimethylation and H3K9 demethylation, is unclear. In any event, the epigenetic 'histone code' switches from transcription-negative $\mathrm{H} 3 \mathrm{~K} 9$ dimethylation to transcription-positive $\mathrm{H} 3 \mathrm{~S} 10$ phosphorylation, and poised promoters eject corepressors NCoR, SMRT, G9a, HP1, HMGB1, and DNMT3a.

The ultimate magnitude of gene expression, including protein production, is also encoded in processes that degrade mRNA - virtually all rapid-response genes are rapidly degraded and contain 3'-UTR AU-rich sequences and/or regulate translation [38]. These mechanisms use RNA-binding proteins and micro-RNAs, whose expression is probably epigenetically controlled.

During the incitement phase of SSI, p65-dependent transcription of autotoxic TNF $\alpha$ and IL- $1 \beta$ genes, among others, peaks within $1-3 \mathrm{~h}$ and then rapidly dissipates, as p65 disappears from the promoter [39]. Gene-encoded proteins also peak and wane. Termination of NFKB-dependent gene transcription requires ubiquitination and proteosome-directed degradation of DNA-bound p65 [33]. Degradation is preceded by deacetylation by NADdependent sirtuin protein 1 [40] coupled to a complex containing copper metabolism Murrl domain-containing proteins (COMM1s) bound to Cullin-RING ubiquitin ligase [41]. Tight regulation is assured by rapid, p65-dependent IкB $\alpha$ transcription and translation in a p65-driven feed-forward loop, providing enough newly synthesized IкB $\alpha$ to resequester p65 and p50. The IкB $\alpha$ promoter as a housekeeping gene is constitutively open in the homeostatic state and further activated during this phase [22]. These cooperative events quickly stop transcription of the acute proinflammatory gene module, producing the precipitous drop in the first-phase curve noted in figure 1 . If the early removal of p 65 is disrupted, transcription is prolonged, and the overall magnitude of gene expression increases SSI risk. Thus, both the height and width of the gene expression curve during the inciting phase of SSI determine the severity of inflammation.

\section{The Evolution Phase Epigenetically Generates Gene-Specific Facultative Heterochromatin to Silence Transcription Selectively and Reversibly}

Before Lewis Thomas colorfully described SSI as 'shambles' [16], Paul Beeson described and presciently interpreted endotoxin tolerance [42], later identified as an 
Table 1. Epigenetic reprogramming in human and murine models of evolving SSI

\begin{tabular}{|c|c|c|c|}
\hline $\begin{array}{l}\text { Transcription repressor/ } \\
\text { activator RelB }\end{array}$ & $\begin{array}{l}\text { de novo synthesis and } \\
\text { promoter binding }\end{array}$ & $\begin{array}{l}\text { de novo synthesis and } \\
\text { promoter binding }\end{array}$ & not reported \\
\hline Histone code switch & $\begin{array}{l}\text { H3S10 phosphorylation to } \\
\text { H3K9 dimethylation }\end{array}$ & $\begin{array}{l}\text { H3S10 phosphorylation to } \\
\text { H3K9 dimethylation }\end{array}$ & $\begin{array}{l}\text { supported by histone deacety- } \\
\text { lation and remodeling complex }\end{array}$ \\
\hline $\begin{array}{l}\text { Gene selection with concomitant } \\
\text { repression and activation }\end{array}$ & yes & yes & yes \\
\hline
\end{tabular}

SSI feature, and now known to be epigenetically based. Comparing Beeson's observations then and now:

Then: requires an inciting pyrogenic inflammatory phase; Now: requires inciting TLR-dependent NFкB p65 transactivation, which induces repressive RelB

Then: dose-dependent phenotypes; Now: gene-expression amplitude induces SIRS vs. SSI

Then: cell-mediated; Now: blood and tissue leukocytemediated

Then: can last for weeks but is reversible; Now: can last for a day (SIRS) or weeks (SSI) but is reversible

Then: a 'remarkable' process with 'no satisfactory mechanism'; Now: a remarkable and SSI-distinct, genespecific, epigenetic reprogramming code

The first nuclear mechanism responsible for the endotoxin tolerance observed in SSI to be discovered was transcriptional repression of multiple genes in circulating blood leukocytes (neutrophils and monocytes/macrophages) $[10,43]$. Another important discovery showed that cellular models of endotoxin tolerance simulate many molecular features of SSI $[44,45]$. 'For over two decades, our studies of the THP1 human promonocytic leukemia cell line have accurately predicted molecular events also observed in SSI blood leukocytes' [3] (table 1).

All SSI phases can be studied in THP1 cells. The genespecific nature of the SSI and endotoxin-tolerance phenotypes, with increased and sustained expression of anti-inflammatory genes coupled to repression of acute pro-inflammatory genes, clinically became known as the compensatory anti-inflammatory syndrome [4]. Its mechanisms are still emerging but involve both transcriptional and posttranscriptional processes. For example, we found mRNA stabilization and enhanced translation of specific genes to be responsible for increased in- terleukin 1 receptor antagonist levels during SSI [46]. This path requires a TLR4-dependent activation of phosphatidyl inositol 3 kinase. Our first clue that epigenetics played a part in SSI was recognizing a nuclear NFкB paradox: as SSI evolves, cytosolic and nuclear activation disassociate [39]. Many animal and human studies reported increased cytosolic NFKB activation with p65 nuclear accumulation during SSI, which correlated with poor prognosis $[3,47]$. This finding seemed contradictory since $\mathrm{NF \kappa B}$-dependent, acute proinflammatory genes are repressed during SSI. To resolve the inconsistency, we analyzed p 65 and p 50 binding at the IL- $1 \beta$ promoter during the transcriptionally silent evolving phase of SSI. We found that transcriptionally repressed leukocytes remained responsive to TLR4 stimulation by endotoxin in THP1 cells and human SSI [48], as shown by rapid degradation of cytosolic Iк $\mathrm{B} \alpha$ and nuclear translocation and accumulation of p65 transcription factor. The change in cytosol signals remains unexplained. We found disrupted p65 promoter binding to IL-1 $\beta$ in the silenced phenotype in both endotoxin-tolerant THP1 cells and human SSI blood leukocytes [39]. In contrast, IL-1 $\beta$ and TNF $\alpha$ promoter binding of p50 occurs in the basal state (discussed above) and during silencing, supporting the conclusion that p50 homodimers participate in silencing acute pro-inflammatory gene expression during endotoxin tolerance and SSI [11, 49].

Our study of disrupted p65 promoter binding prompted epigenetic analysis of the evolving phase of SSI associated with endotoxin tolerance. We found that an activated histone code for $\mathrm{H} 310$ phosphorylation switches to repressive H3K9 dimethylation during the incitement phase [39]. This was the first hint that an epigenetic paradigm plays a crucial role in regulating gene expression during SSI. 
We later implicated RelB as a negative regulator both necessary and sufficient for silencing acute proinflammatory IL-1 $\beta$ and TNF $\alpha$ during endotoxin tolerance and human SSI [48]. This unique NFкB family member was first characterized as an NF $\kappa \mathrm{B}$ repressor and then as a positive transcription factor that primarily heterodimerizes with NFкB p100 or p52 [50]. RelB-deficient mice have generalized multiorgan inflammation [51]. RelB protein, which is not expressed in the basal homeostatic phase in innate immunity leukocytes, is induced as SSI evolves. Once it accumulates in the nucleus, RelB acts in a p65-dependent feed-forward loop to repress specific gene sets (fig. 2). It binds coincident retained p50 on the IL-1 $\beta$ and TNF $\alpha$ proximal promoters in a presumed dimer switch with p65 [52], but also accumulates with promoter p50 to activate IкB $\alpha$ transcription. RelB removal by siRNA reverses facultative heterochromatin and restores IL-1 $\beta$ transcription in both THP1 cells and human SSI leukocytes but inhibits ІкB $\alpha$ transcription $[22,53]$. Transfection analysis of wild-type RelB into naïve THP1 cells further establishes it as a crucially important dual regulator. The transfected RelB requires a TLR4 signal to move to promoters.

RelB initiates silent facultative heterochromatin at the IL-1 $\beta$ and TNF $\alpha$ promoters by forming a complex of HP1 and G9a H3K9 dimethyl transferase [21]. The Rel homology domain directly interacts with the $\mathrm{N}$ terminal of G9a, which generates the histone $\mathrm{H} 3 \mathrm{~K} 9$ dimethylated silent state. HP1 binds to methylated sites, and siRNA RelB knockdown prevents formation of the facultative heterochromatin complex and transcription silencing. Together, our data support the conclusion that two feed-forward loops involving p65 and RelB target both TNF $\alpha$ and IL$1 \beta$ as a silenced module and IкB $\alpha$ as part of an activated module (fig. 2).

We identified more components of the silencing chromatin complex associated with evolving SSI. HP1 recruitment also links to DNA methyl transferase Dnmt3a/b, which de novo methylates TNF $\alpha$ promoter CpG [27]. Other reports indicate that Dnmt3a/b associates with SUV39H1 histone methyltransferase, which trimethylates H3K27 and recruits polycomb group proteins to propagate condensed chromatin [2]. We have not studied polycomb group proteins, but SUV39H1 is recruited to the TNF $\alpha$ promoter after G9a and HP1 [27].

Nuclear structural proteins also play an important role in epigenetic silencing and formation of facultative heterochromatin. Histone 1 (H1) linker protein accumulates at repressed promoters, targeting the DNA entry site [54]. Both $\mathrm{H} 1$ and high-mobility group B1

Epigenetics of Infection
(HMGB1) histone structural protein bind to linker DNA near the nucleosome dyad. In some studies, these two structural proteins have opposing effects on the nucleosome: H1 stabilizes it by 'sealing' two turns of DNA around the histone octamer, and HMGB1 destabilizes it, probably by bending the adjacent DNA. We find both $\mathrm{H} 1$ and HMGB1 in condensed TNF $\alpha$ chromatin during the SSI evolving phase [53]. We also made the unexpected observation that HMGB1 acts as a repressor. If HMGB1 and $\mathrm{H} 1$ are removed by siRNA, RelB does not bind to the $\mathrm{TNF} \alpha$ promoter and facultative heterochromatin does not form. Thus, these two structural determinants play a crucial role in initiating RelB function and may place a temporary lock on facultative heterochromatin through $\mathrm{H} 1$. Interestingly, we do not find $\mathrm{H} 1$ at the poised basal promoter. Interestingly, as a cooperative nuclear repressor in SSI, HMGB1 acts as both a negative and positive regulator of inflammation when located extracellularly.

Together, our data support the conclusion that RelB and G9a combine to foster a large, interdependent, multimolecular epigenetic complex that includes histone modifiers, DNA methyl transferases, and chromatin structural proteins (fig. 2). Other TLR-induced transcription repressor proteins induced during the evolving SSI phase that might directly participate in the epigenetic silencing have been identified. These include inducible cAMP early repressor (ICER) that binds to CRE sites [55], B cell lymphoma 3 (BCL3) that binds to $\mathrm{p} 50$ to block its proteosome degradation [56], and ATF3 that negatively regulates CRE sites [19]. No reports to our knowledge have performed detailed epigenetic analysis linked to the repressors, nor whether they are a part of the RelB program.

\section{The SSI Resolution Phase Correlates with Disappearance of the Feed-Forward Loops and Resumption of the p65-Dependent Incitement Phase}

Little is understood about epigenetic events in the resolution stage of SSI, except how important they are. Reversal of facultative heterochromatin to euchromatin portends rescue and survival - the end of shambles and endotoxin tolerance. Building an understanding of genespecific reprogramming in cell models, animal models, and human SSI is therefore critical.

The course of epigenetic, gene-specific silencing varies. Humans given a low dose of intravenous endotoxin generate endotoxin tolerance, which is sustained for 
about $24 \mathrm{~h}$ [57], when blood leukocytes again respond to TLR signals. This appears typical of SIRS. Gene-selective reprogramming can last much longer, up to several weeks in human SSI [10]. This 'memory' supports but does not prove that vertically passed epigenetics occurs in shortlived blood and tissue innate immune cells (e.g. neutrophils). The duration of the silencing phase in humans or animals with SSI probably varies with the magnitude of the threat, which informs chromatin how long to sustain the gene-specific facultative heterochromatin. The type of infecting organism has little effect on the stereotypic SSI process [5].

The resolution phase in the THP1 model provides an excellent way to follow the return of facultative heterochromatin to poised euchromatin. Our preliminary data support the conclusion that after $72-96 \mathrm{~h}$, RelB protein levels wane, and RelB promoter binding diminishes. The cells regain their ability to bind p65 and incite transcription, although it cannot be reinduced without endotoxin stimulation of TLR4.

\section{Understanding the Concepts Preventing versus Reversing Endotoxin Tolerance/SSI Epigenetic Gene-Selective Reprogramming Is Critical to Improving SSI Therapy}

Facultative heterochromatin, by definition, is a reversible structure, unlike pericentric heterochromatin [58]. A number of reports propose reversing endotoxin tolerance. However, this interpretation is often flawed: gene silencing/tolerance were prevented, not reversed. Since silencing requires a $\mathrm{p} 65$-dependent inciting phase, any manipulation that disrupts NFкB cytosolic or nuclear processes will prevent it. Moreover, removing a constitutive negative homeostatic regulator prior to the inciting phase increases transcription magnitude, systemic inflammation, and, consequently, mortality in animal models. This prevention paradigm likely applies to all de novo repressors implicated in endotoxin tolerance, including $\mathrm{SH} 2$ domain polyinositol-5-phosphatase-1, IL-1 receptor-associated kinase 1-M, and A20 zinc finger editor of ubiquitin RelB, ICER , BCL3, and ATF3 [3, 17]. Whether removal of these proteins after chromatin condenses alters or reverses silencing is a very different question.

We do find that facultative heterochromatin can be reversed after it is condensed. For example, RelB siRNA delivered during the silencing phase and the protein synthesis inhibitor cycloheximide (which produces RelB protein degradation) reverse the facultative heterochro- matin state and restore p65-dependent transcription [submitted for publication]. However, reversing silencing to induce resolution is not simple. Inhibition of 'classical' class 1 and 2 (HDAC) by trichostatin enhances the incitement phase but can neither block the development of tolerance nor restore endotoxin sensitivity in the evolution phase [59]. In contrast, the protein phosphatase 1 inhibitor okadaic acid stabilizes IL-1 $\beta$ mRNA to increase protein production in transcriptionally silenced cells but cannot activate transcription [60], supporting the conclusions that both transcription and posttranscription controls regulate gene expression during SSI. More work is needed to discover ways to reverse SSI gene-specific repression.

\section{Nuclesomes Reposition during Each SSI Phase}

Promoter nucleosomes adopt certain positions to regulate transcription factor binding and are displaced from promoter DNA by promoter-binding transcription factors, chromatin-remodeling complexes, and histone chaperones [61]. The selective nucleosomal organization observed in many systems provides different access to chromatin structure and different transcriptional potential between active and inactive promoters.

We recently mapped the nucleosomes on the silenced $\mathrm{TNF} \alpha$ proximal promoter in the endotoxin-tolerant THP1 SSI model [29]. Two proximal nucleosomes reposition from the basal poised state to a permissive location during the lipopolysaccharide-inciting stage, and move to repressive locations in the evolution stage (fig. 3). This sequence prevents, allows, and then again prevents access to p65 during the distinct phases. Moreover, ectopic expression of RelB induction during the evolution phase promotes nucleosome repositioning to repressive locations, while RelB inhibition in tolerant cells moves the same nucleosomes to permissive locations (fig. 3). This shifting supports reformation of a poised state during resolution, although the nucleosomes' exact locations may differ from basal homeostasis.

We also found that the ATP-independent chromatin remodeler NAP1 promotes nucleosome positioning during incitement [29]. In contrast, nucleosome repositioning during the formation of facultative heterochromatin requires the ATP-dependent BAF member of the SWI/ SNF remodeling complex. Together, these results support the conclusion that TNF $\alpha$ promoter nucleosomes selectively and dynamically remodel during SSI phases. 


\section{The Epigenetic Human SSI Paradigm Occurs in Mice}

Our investigations of SSI epigenetics exclusively involve human cells. An elegant study using macrophages derived from murine bone-marrow confirms and extends our understanding of epigenetic gene-specific regulation [15]. Using an in vitro system of lipopolysaccharide tolerance, two functionally distinct classes of TLR4induced genes required distinct patterns of TLR-induced chromatin modifications consistent with our studies in THP1 cells and human SSI. Putative facultative heterochromatin was coupled with transient silencing of acute proinflammatory mediators and activation of antimicrobial and anti-inflammatory mediators. The functions of RelB in initiating repression and activating transcription were not investigated. Table 1 compares gene reprogramming features of THP1 cells, murine macrophages, and human SSI. It is suspected, but not proven, that murine SSI in vivo will simulate the epigenetic paradigm.

\section{Perspective}

Our understanding of epigenetic regulation of SSI is emerging, and further defining its features will inform basic biology, translational science, and clinical medicine. Observations and related questions include:

(1) New approaches to SSI intervention are needed. For example, (1) Will reversing facultative heterochromatin during the evolution stage alter the outcome? (2) Do the two interventions (anti-HMGB1 and ethyl pyruvate) found to improve outcomes when administered during evolution modify gene reprogramming?

(2) The new signaling networks generated by gene reprogramming must be determined. What alternative TLR4-dependent paths activate cytosolic NFкB during the evolution phase?

(3) The spatial and temporal features of chronic and acute inflammation vary. How does the epigenetic process of chronic inflammation, which does not disrupt p65 binding to silence acute proinflammatory genes, differ from SSI?

(4) NF $B$ nuclear disruption with gene silencing may differ among compartments (e.g. liver vs. lung). Do such compartments have tissue-specific gene reprogramming?

(5) SSI represses and activates genes. What global changes in transcription and translation couple with the two RelB-dependent feed-forward loops? Do other proteins that act to negatively regulate transcription at the level of DNA (e.g. ATF3, ICER, Bcl3) act with or dis- tinct from RelB? What epigenetic processes sustain activation? What proximal signaling networks influence each phase of SSI?

(6) ATP depletion and mitochondrial damage occur during the SSI incitement phase. Is cellular energy linked to epigenetic regulation?

(7)Micro RNAs help to regulate inflammation. Do they influence SSI phases?

(8) SSI resolution correlates with the reversal of silencing facultative heterochromatin. What processes restore poised euchromatin?

\section{Conclusions}

An epigenetic dynamic between proteins and chromatin histones and DNA instructs gene transcription during the inciting, evolving, and resolving phases of inflammation. In serious infections with SSI, the inciting phase is like any other, except for its magnitude: TLRdependent signals use the NFKB master regulator to rapidly derepress, activate and quickly deactivate the epigenetically poised promoters of acute proinflammatory genes. The evolving phase of SSI, however, is distinct: feed-forward loops generated by the inciting phase epigenetically silence or activate selective genes, according to function. To silence acute proinflammatory gene transcription - a state of endotoxin tolerance - de novo induction of RelB recruits an interdependent multicomponent facultative heterochromatin complex that repositions proximal promoter nucleosomes. To activate anti-inflammatory gene transcription, dual functioning RelB recruits to responsive euchromatin. These epigenetic modifications revert as SSI resolves and homeostasis returns. Thus, epigenetics phenotypically delineate the clinical features of SSI.

\section{Acknowledgments}

We thank Richard Loeser for reviewing the manuscript, Sue Cousart and Jean Hu for technical assistance. This work was partially supported by NIH grants: RO1 AI-09169 (cem), RO1 AI065791 (cem), R01 AI-079144 (cem), and MO-1RR 007122 (WFU General Clinical Research Center). 


\section{References}

1 Majno, G: The Healing Hand. Man and Wound Healing. Boston, Harvard Press, 1975.

$\checkmark 2$ Kouzarides T: Chromatin modifications and their function. Cell 2007;128:693-705.

$\checkmark 3$ McCall CE, Yoza BK: Gene silencing in severe systemic inflammation. Am J Respir Crit Care Med 2007;175:763-767.

-4 Bone RC: Sir Isaac Newton, sepsis, SIRS, and CARS. Crit Care Med 1996;24:1125-1128.

5 Hotchkiss RS, Karl IE: The pathophysiology and treatment of sepsis. N Engl J Med 2003; 348:138-150.

6 Medzhitov R, Horng T: Transcriptional control of the inflammatory response. Nat Rev Immunol 2009;9:692-703.

$\checkmark 7$ Schreiber J, Jenner RG, Murray HL, Gerber GK, Gifford DK, Young RA: Coordinated binding of NF- $\mathrm{KB}$ family members in the response of human cells to lipopolysaccharide. Proc Natl Acad Sci USA 2006;103:58995904.

$>8$ Takeda K, Akira S: TLR signaling pathways. Semin Immunol 2004;16:3-9.

$>9$ Ghisletti S, Huang W, Jepsen K, et al: Cooperative NCoR/SMRT interactions establish a corepressor-based strategy for integration of inflammatory and anti-inflammatory signaling pathways. Genes Dev 2009;23:681693.

$>10$ McCall CE, Grosso-Wilmoth LM, LaRue K, Guzman RN, Cousart SL: Tolerance to endotoxin-induced expression of the interleukin$1 \beta$ gene in blood neutrophils of humans with the sepsis syndrome. J Clin Invest 1993;91: 853-861.

$\checkmark 11$ Mueller LP, Yoza BK, Neuhaus K, et al: Endotoxin-adapted septic shock leukocytes selectively alter production of sIL-1RA and IL$1 \beta$. Shock 2001;16:430-437.

12 West MA, Heagy W: Endotoxin tolerance: a review. Crit Care Med 2002;30(suppl):S64S73.

-13 Feldmann M, Maini RN: Lasker Clinica Medical Research Award. TNF defined as a therapeutic target for rheumatoid arthritis and other autoimmune diseases. Nat Med 2003;9:1245-1250.

14 Suarez Y, Wang C, Manes TD, Pober JS: Cutting edge: TNF-induced microRNAs regulate TNF-induced expression of E-selectin and intercellular adhesion molecule-1 on human endothelial cells: feedback control of inflammation. J Immunol 2010;184:21-25.

-15 Foster SL, Hargreaves DC, Medzhitov R: Gene-specific control of inflammation by TLR-induced chromatin modifications. Nature 2007;447:972-978.

16 Thomas L: The Lives of a Cell. New York, Viking Press, 1974.

17 Liew FY, Xu D, Brint EK, O’Neill LA: Negative regulation of Toll-like receptor-mediated immune responses. Nat Rev Immunol 2005;5:446-458.
18 Zak DE, Aderem A: A systems view of host defense. Nat Biotechnol 2009;27:999-1001.

19 Gilchrist M, Thorsson V, Li B, et al: Systems biology approaches identify ATF3 as a negative regulator of Toll-like receptor 4 . Nature 2006;441(7090):173-178

20 Alon U: Network motifs: theory and experimental approaches. Nat Rev Genet 2007;8 450-461.

21 Chen X, El GM, Yoza BK, McCall CE: The $\mathrm{NF}-\kappa \mathrm{B}$ factor RelB and histone $\mathrm{H} 3$ lysine methyltransferase G9a directly interact to generate epigenetic silencing in endotoxin tolerance. J Biol Chem 2009;284:2785727865.

22 Chen X, Yoza BK, El GM, Hu JY, Cousart SL, McCall CE: RelB sustains $\mathrm{I} \kappa \mathrm{B} \alpha$ expression during endotoxin tolerance. Clin Vaccine Immunol 2009;16:104-110.

23 Aderem A, Smith KD: A systems approach to dissecting immunity and inflammation. Semin Immunol 2004;16:55-67.

24 Perissi V, Jepsen K, Glass CK, Rosenfeld MG: Deconstructing repression: evolving models of co-repressor action. Nat Rev Genet 2010; 11:109-123.

25 Hargreaves DC, Horng T, Medzhitov R: Control of inducible gene expression by signal-dependent transcriptional elongation. Cell 2009;138:129-145.

26 Huang W, Ghisletti S, Perissi V, Rosenfeld MG, Glass CK: Transcriptional integration of TLR2 and TLR4 signaling at the NCoR derepression checkpoint. Mol Cell 2009;35:4857.

27 El GM, Yoza BK, Chen X, Hu J, Hawkins GA, McCall CE: G9a and HP1 couple histone and DNA methylation to TNF $\alpha$ transcription silencing during endotoxin tolerance. J Biol Chem 2008;283:32198-32208

28 Izzo A, Kamieniarz K, Schneider R: The histone $\mathrm{H} 1$ family: specific members, specific functions? Biol Chem 2008;389:333-343.

29 El GM, Liu T, Yoza BK, McCall CE: Dynamic and selective nucleosome repositioning during endotoxin tolerance. J Biol Chem 2010;285:1259-1271.

30 Kruys V, Thompson P, Beutler B: Extinction of the tumor necrosis factor locus, and of genes encoding the lipopolysaccharide signaling pathway. J Exp Med 1993;177:13831390.

31 Foster SL, Medzhitov R: Gene-specific control of the TLR-induced inflammatory response. Clin Immunol 2009;130:7-15.

32 El GM, Yoza BK, Hu JY, Cousart SL, McCall $\mathrm{CE}$ : Epigenetic silencing of tumor necrosis factor- $\alpha$ during endotoxin tolerance. J Biol Chem 2007;282:26857-26864.

33 Saccani S, Marazzi I, Beg AA, Natoli G: Degradation of promoter-bound p65/RelA is essential for the prompt termination of the nuclear factor $\kappa \mathrm{B}$ response. J Exp Med 2004; 200:107-113.
34 Maine GN, Burstein E: COMMD proteins and the control of the NF $\kappa \mathrm{B}$ pathway. Cell Cycle 2007;6:672-676.

35 Riedemann NC, Guo RF, Ward PA: The enigma of sepsis. J Clin Invest 2003;112:460467.

36 Akira S: Toll-like receptors: lessons from knockout mice. Biochem Soc Trans 2000;28: 551-556.

37 Hoffmann A, Baltimore D: Circuitry of nuclear factor $\mathrm{\kappa B}$ signaling. Immunol Rev 2006;210:171-186.

38 Hao S, Baltimore D: The stability of mRNA influences the temporal order of the induction of genes encoding inflammatory molecules. Nat Immunol 2009;10:281-288.

>39 Chan C, Li L, McCall CE, Yoza BK: Endotoxin tolerance disrupts chromatin remodeling and NF-kappaB transactivation at the IL$1 \beta$ promoter. J Immunol 2005; 175:461-468.

-40 Yeung F, Hoberg JE, Ramsey CS, et al: Modulation of NF- $\mathrm{B}$-dependent transcription and cell survival by the SIRT1 deacetylase. EMBO J 2004;23:2369-2380.

41 Maine GN, Mao X, Muller PA, Komarck CM, Klomp LW, Burstein E: COMMD1 expression is controlled by critical residues that determine XIAP binding. Biochem J 2009;417: 601-609.

42 Beeson P: Tolerance to bacterial pyrogens. J Exp Med 1947;86:29-41.

43 Munoz C, Carlet J, Fitting C, Misset B, Bleriot JP, Cavaillon JM: Dysregulation of in vitro cytokine production by monocytes during sepsis. J Clin Invest 1991;88:1747-1754.

44 Larue KEA, McCall CE: A labile transcriptional repressor modulates endotoxin tolerance. J Exp Med 1994;180:2269-2275.

45 Virca GD, Kim SY, Glaser KB, Ulevitch RJ Lipopolysaccharide induces hyporesponsiveness to its own action in RAW 264.7 cells. J Biol Chem 1989;264:21951-21956.

46 Learn CA, Boger MS, Li L, McCall CE: The phosphatidylinositol 3-kinase pathway selectively controls sIL-1RA not interleukin$1 \beta$ production in the septic leukocytes. J Biol Chem 2001;276:20234-20239.

47 Bohrer H, Qiu F, Zimmermann T, et al: Role of NFKB in the mortality of sepsis. J Clin Invest 1997;100:972-985.

-48 Yoza BK, Hu JY, Cousart SL, Forrest LM, McCall CE: Induction of RelB participates in endotoxin tolerance. J Immunol 2006;177: 4080-4085.

49 Bohuslav J, Kravchenko VV, Parry GCN, et al: Regulation of an essential innate immune response by the $\mathrm{p} 50$ subunit of NF- $\mathrm{\kappa B}$. J Clin Invest 1998;102:1645-1652.

50 Ryseck RP, Weih F, Carrasco D, Bravo R: RelB, a member of the Rel/NF- $\kappa$ B family of transcription factors. Braz J Med Biol Res 1996;29:895-903. 
51 Weih F, Carrasco D, Durham SK, et al: Multiorgan inflammation and hematopoietic abnormalities in mice with a targeted disruption of RelB, a member of the NF- $\kappa \mathrm{B} / \mathrm{Rel}$ family. Cell 1995;80:331-340.

52 Saccani S, Pantano S, Natoli G: Modulation of NF- $\mathrm{KB}$ activity by exchange of dimers. Mol Cell 2003;11:1563-1574.

53 El GM, Yoza BK, Chen X, Garcia BA, Young NL, McCall CE: Chromatin-specific remodeling by HMGB1 and linker histone $\mathrm{H} 1$ silences proinflammatory genes during endotoxin tolerance. Mol Cell Biol 2009;29: 1959-1971.

-54 Rupp RA, Becker PB: Gene regulation by histone H1: new links to DNA methylation. Cell 2005;123:1178-1179.
55 Harzenetter MD, Novotny AR, Gais P, Molina CA, Altmayr F, Holzmann B: Negative regulation of TLR responses by the neuropeptide CGRP is mediated by the transcriptional repressor ICER. J Immunol 2007;179: 607-615.

56 Carmody RJ, Ruan Q, Palmer S, Hilliard B, Chen YH: Negative regulation of Toll-like receptor signaling by NF- $\mathrm{\kappa B}$ p50 ubiquitination blockade. Science 2007;317(5838):675678.

57 van der Poll T, Coyle SM, Moldawer LL, Lowry SF: Changes in endotoxin-induced cytokine production by whole blood after in vivo exposure of normal humans to endotoxin. J Infect Dis 1996;174:1356-1360.
58 Trojer P, Reinberg D: Facultative heterochromatin: is there a distinctive molecular signature? Mol Cell 2007;28:1-13.

59 Yoza BK, Hu JY, McCall CE: Inhibition of histone deacetylation enhances endotoxinstimulated transcription but does not reverse endotoxin tolerance. J Endotoxin Res 2002; 8:109-114.

60 Yoza BK, Wells JD, McCall CE: Interleukin$1 \beta$ expression after inhibition of protein phosphatases in endotoxin-tolerant cells. Clin Diagn Lab Immunol 1998;5:281-287.

61 Segal MR: Re-cracking the nucleosome positioning code. Stat Appl Genet Mol Biol 2008; 7:Article14. 